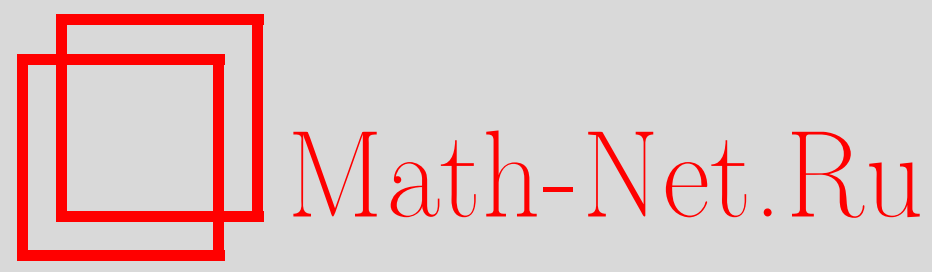

А. М. Чеботарев, Квантовое стохастическое уравнение унитарно эквивалентно симметричной краевой задаче для уравнения Шредингера, Матем. заметки, 1997, том 61, выпуск 4, 612-622

DOI: https://doi.org/10.4213/mzm1539

Использование Общероссийского математического портала Math-Net.Ru подразумевает, что вы прочитали и согласны с пользовательским соглашением http://www.mathnet.ru/rus/agreement

Параметры загрузки:

IP: 54.174 .149 .18

26 апреля 2023 г., 16:32:59

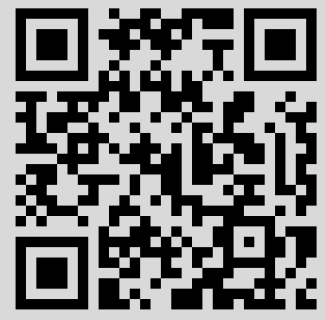




\title{
КВАНТОВОЕ СТОХАСТИЧЕСКОЕ УРАВНЕНИЕ УНИТАРНО ЭКВИВАЛЕНТНО СИММЕТРИЧНОЙ КРАЕВОЙ ЗАДАЧЕ ДЛЯ УРАВНЕНИЯ ШРЁДИНГЕРА
}

\section{А. М. Чеботарев}

\begin{abstract}
Показано, что решение квантового стохастического уравнения Хадсона-Партасарати в фоковском пространстве совпадает с решением симметричной краевой задачей для уравнения Шрёдингера в представлении взаимодействия, порождаемом оператором энергии окружения. Краевые условия описывают скачки фазы и амплитуды Фурье-образов компонент фоковского вектора при перемене знака любого из его аргументов. Соответствующее уравнение марковской эволюции (уравнение Линдблада или "master equation") выводится исходя из краевой задачи для уравнения Шрёдингера.

Библиография: 28 названий.
\end{abstract}

1. Введение. Наш интерес к теории квантовых стохастических дифференциальных уравнений связан прежде всего с возможностью представить с их помошю решения уравнения марковской эволюции, обобщающего уравнения Гейзенберга, Колмогорова-Феллера и уравнение теплопроводности [1]-[4].

Решения уравнений марковской эволюции изучаются в теории открытых квантовых систем [5]-[8] и назьваются квантовыми динамическими полугруппами. Одним из следствий стохастического представления квантовых динамических полугруп в алгебрах фон Неймана является простая связь между свойством консервативности полугруппы и изометричностью решения квантовых стохастических дифференциальных уравнений. Консервативность (или унитальность) квантовой динамической полугруппы означает сохранение единищы операторной алгебры и соответствует сохранению полной вероятности решениями уравнений для переходных вероятностей случайных процессов в классическом и квантовом случаях. Нарушение этого свойства связано с критическими явлениями типа взрыва, ухода решения на бесконечность, накопления бесконечного числа точек разрыва, неоднозначностью решения задачи Коши, наличием растущих решений у формально диссипативных уравнений и т.п. [9], [10]. Изучение необходимых и достаточных условий возникновения или отсутствия таких явлений представляет большой интерес [11]-[18].

В последние годы в основном пертурбативньми методами были получены достаточные критерии изометричности и унитарности решений квантовых динамических полугрупп [19]-[21]. Однако, не были найдены симметричные операторы, ответственные за унитарность и изометричность решений квантовых стохастических дифференциальных

Работа выполнена при финансовой поддержке Российского фонда фундаментальных исследований, грант № 95-01-00784. 
уравнений. Трудности на этом пути сводятся, во-первых, к отсутствию группового свойства решений (см. [22]) и, во-вторых, к несимметричности формальных генераторов на областях определения, состоящих из гладких функций. Более точно (см. [23]), формальные генераторы уравнений Шрёдингера, унитарно эквивалентных квантовым стохастическим дифференциальным уравнениям, имеют вид диссипативньх операторов, возмущенных сингулярньпи в смысле [24], [25] квадратичными формами. Наличие неограниченной антиэрмитовой части у диссипативной составляющей генератора затрудняет использование общей теории, развитой для построения самосопряженных расширений ограниченньх снизу симметричных операторов, возмушенных сингулярными квадратичными формами [24]-[26].

В настоящей работе рассматриваются квантовые стохастические дифференциальные уравнения, решения которых в представлении взаимодействия являются пределами решений семейства уравнений Шрёдингера в фоковском пространстве [23]. Принципиальным свойством резольвенты предельной унитарной группы является наличие скачков амплитуды и фазы у функций из области ее значений в точках сингулярности квадратичной формы генератора предельной унитарной группы. Вычисляя действие генератора грушы на множестве функций с разрывами амплитуды и фазы, мы получаем симметричный оператор, самосопряженность которого проверяется независимым образом при менее ограничительных предположениях, чем те, которые используются при явном построении резольвенты. Таким образом, доказывается эквивалентность квантового стохастического дифференциального уравнения и краевой задачи для уравнения Шрёдингера в фоковском пространстве. Прямые вычисления позволяют вывести уравнение марковской эволюции непосредственно из краевой задачи для уравнения Шрёдингера.

Начнем с простого примера, демонстрирующего различие между слабым формальным пределом, сильным резольвентным пределом последовательности генераторов унитарных групп и действием генератора предельной унитарной группы на множестве значений резольвенты.

2. Слабый и резольвентный пределы семейства генераторов унитарных однопараметрических групп. Рассмотрим семейство унитарных групा $\exp \left\{i t \widehat{H}_{\alpha}\right\}=U_{t}^{(\alpha)}$, действуюших в $L_{2}(\mathbb{R})$ :

$$
U_{t}^{(\alpha)} \psi(x)=\psi(x-t) \exp \left\{i \lambda \int_{0}^{t} d \tau V_{\alpha}(x-t+\tau)\right\}, \quad x, \lambda \in \mathbb{R},
$$

где $V_{\alpha}(x)=(2 \pi \alpha)^{-1 / 2} \exp \left\{-x^{2} / 2 \alpha\right\}, \alpha \in \mathbb{R}_{+}$. Очевидно, что $V_{\alpha}(x) \rightarrow \delta(x)$ при $\alpha \rightarrow+0$. Поэтому слабый предел семейства существенно самосопряженных операторов $\widehat{H}_{\alpha}=i \partial_{x}+\lambda V_{\alpha}(x)$ описывается билинейной формой

$$
\widehat{H}_{w}[\varphi, \psi]=\left(\varphi, \widehat{H}_{w} \psi\right)=i\left(\varphi, \psi^{\prime}\right)+\lambda \bar{\varphi}(0) \psi(0),
$$

корректно определенной на функциях из $W_{2}^{1}(\mathbb{R})$.

С другой стороны,

$$
\int_{0}^{t} d \tau V_{\alpha}(x-t+\tau) \rightarrow I_{[0, t)}(x)
$$

где $I_{T}(x)$ - индикаторная функция борелевского множества $T \subseteq \mathbb{R}$. Следовательно, сильный предел семейства унитарных групп равен

$$
\lim _{\alpha \rightarrow+0} U_{t}^{(\alpha)} \psi(x)=U_{t} \psi(x)=e^{i t \widehat{\mathbf{H}}_{R}} \psi(x)=\psi(x-t) e^{i \lambda I_{[0, t)}(x)} .
$$


Заметим, что имеет место тождество $e^{i \lambda I_{[0, t)}(x)}=\left(e^{i \lambda}-1\right) I_{[0, t)}(x)+1$. Поэтому билинейная форма резольвентного предела $\widehat{\mathbf{H}}_{R}=r-\lim \widehat{H}_{\alpha}$ корректно определена на $W_{2}^{1}(\mathbb{R})$ и действует по правилу

$$
\widehat{\mathbf{H}}_{R}[\varphi, \psi]=\left.i^{-1} \lim _{t \rightarrow 0} \frac{d}{d t}\left(\varphi, U_{t} \psi\right)\right|_{t=0}=i\left(\varphi, \psi^{\prime}\right)+i\left(e^{i \lambda}-1\right) \bar{\varphi}(0) \psi(0) .
$$

Сравнивая (2.1) и (2.2), получаем $\widehat{\mathbf{H}}_{R}=r-\lim \widehat{H}_{\alpha} \neq w-\lim H_{\alpha}=\widehat{H}_{w}$.

Множество значений резольвенты предельной унитарной группы $U_{t}$ является естественной областью определения генератора $\widehat{\mathbf{H}}_{R}$ и в рассматриваемом примере может быть описано явно:

$$
R_{\mu} \psi(x)=\int_{0}^{\infty} d t e^{-\mu t} \psi(x-t)+\theta(x)\left(e^{i \lambda}-1\right) e^{-\mu x} \int_{0}^{\infty} d t e^{-\mu t} \psi(-t),
$$

где $\theta(x)$ - индикаторная функция полупрямой $\mathbb{R}_{+}$. Такая структура резольвенты указьвает на то, что функции из области определения еегенератора $\widehat{\mathbf{H}}_{R}$ имеют скачок фазы при $x=0$ :

$$
\lim _{x \rightarrow+0} R_{\mu} \psi(x)=e^{i \lambda} \lim _{x \rightarrow-0} R_{\mu} \psi(x) .
$$

Следовательно, область определения $\mathscr{D}_{\lambda}$ генератора $\widehat{\mathbf{H}}_{R}$ состоит из функций

$$
\psi: \quad \psi \in W_{2}^{1}(\mathbb{R} \backslash\{0\}), \quad \lim _{x \rightarrow+0} \psi(x)=e^{i \lambda} \lim _{x \rightarrow-0} \psi(x) .
$$

При $x \neq 0$ оператор $\widehat{\mathbf{H}}_{R}$ действует как $i \partial_{x}$. Левьй и правый пределы в нуле для функций из $\mathscr{D}_{\lambda}$ существуют в силу вложения $W_{2}^{1}(\mathbb{R} \backslash\{0\}) \subset C(\mathbb{R} \backslash\{0\})$. Симметричность оператора $\widehat{\mathbf{H}}_{R}$ следует из формулы интегрирования по частям и равенства

$$
\left.\overline{\varphi(x)} \psi(x)\right|_{+0} ^{-0}=0
$$

для функций из $\mathscr{D}_{\lambda}$, а его самосопряженность вытекает (см. [27]) из разрешимости в $\mathscr{D}_{\lambda}$ уравнения $\left(\widehat{\mathbf{H}}_{R}+i \mu\right) \psi(x)=f(x), x \neq 0$, с граничным условием (2.3) для любой правой части из $L_{2}(\mathbb{R})$ при $\mu= \pm 1$.

\section{3. Слабый и сильный резольвентный пределы решений уравнения Шрё-} дингера в фоковском пространстве. Пусть $\mathscr{H}$ - гильбертово пространство, $\Gamma^{S}\left(L_{2}(\mathbb{R})\right)$ - симметричное фоковское пространство и $\mathbf{h}=\mathscr{H} \otimes \Gamma^{S}\left(L_{2}(\mathbb{R})\right)$-их тензорное произведение. Для $v, g \in L_{2}(\mathbb{R})$ обозначим через $A^{+}(v), A(g)$ стандартные операторы рождения и уничтожения в $\Gamma^{S}\left(L_{2}(\mathbb{R})\right)$ и рассмотрим семейство уравнений Шрёдингера $\partial_{t} \psi_{t}=i H \psi_{t}$ с самосопряженным в $\mathbf{h}$ гамильтонианом $\mathbf{H}=H_{0} \otimes I+I \otimes \widehat{E}+H_{\mathrm{int}}$ :

$$
H_{\mathrm{int}}=K \otimes A^{+}(g) A(g)+R \otimes A^{+}(f)+R^{*} \otimes A(f), \quad \widehat{E}=\int \omega a^{+}(\omega) a(\omega) d \omega .
$$

Для упрощения в дальнейшем предполагается, что операторы $H_{0}, K, R \in \mathscr{C}(\mathscr{H})$ коммутируют и имеют общее спектральное семейство $E_{\lambda}$. Более точно, пусть

$$
H_{0}=\int \nu(\lambda) d E_{\lambda}, \quad K=\int \lambda d E_{\lambda}, \quad R=\int \rho(\lambda) e^{i \Phi(\lambda)} d E_{\lambda},
$$


где $\nu, \rho, \Phi$ - измеримые вещественные функции, соответствующие операторам $H_{0}, K, R$, т.е. операторы $H_{0}, K$ самосопряженные, а $R$ нормальньй.

Обозначим через $\widehat{P}_{t}(\lambda)$ однопараметрическую групу унитарных операторов в $L_{2}(\mathbb{R})$ с генератором $\widehat{N}(\lambda)=\omega+\lambda|g\rangle\langle g|$. Унитарная группа $U_{t}=\exp \{i \mathbf{H} t\}$ может быть построена методом преобразования гамильтониана к каноническому виду (см. [28]) либо с помощью использования представления взаимодействия, порождаемого оператором $I \otimes \widehat{E}+K \otimes A^{+}(g) A(g)$ (см. [23]). Теорема 3.1 описывает действие $U_{t}$ на когерентные векторы.

ТЕоремА 3.1. Унитарная однопараметрическая группа операторов $U_{t}=$ $\exp \{i t \mathbf{H}\}$, əде $\mathbf{H}=H_{0} \otimes I+I \otimes \widehat{\mathbf{E}}+H_{\mathrm{int}}$, a оператор $H_{\mathrm{int}}$ определен формулой $(3.1)$, действует по правилу

$$
U_{t} h \otimes \psi(v)=\int e^{i \nu(\lambda) t} d E_{\lambda} h \otimes \psi\left(v_{t}(\lambda)\right) \exp \left\{i \rho(\lambda) e^{-i \Phi(\lambda)} \int_{0}^{t}\left(f, v_{s}(\lambda)\right) d s\right\}
$$

$2 \partial e$

$$
v_{t}(\lambda)=\widehat{P}_{t}(\lambda) v+i \rho(\lambda) e^{i \Phi(\lambda)} \int_{0}^{t} \widehat{P}_{s}(\lambda) f d s .
$$

Обозначим через $L_{2, \widetilde{1}}^{+}(\mathbb{R})$ множество функщий из $\widetilde{W}_{2}^{1}(\mathbb{R})$, имеющих абсолютно интегрируемый положительньй $Ф$ уье-образ, $\widetilde{W}_{2}^{1}(\mathbb{R})$ - Фурье-образ соболевского пространства $W_{2}^{1}(\mathbb{R})$. Пусть $f, g \in L_{2, \tilde{1}}^{+}(\mathbb{R})$ - такие вещественные функции, что $f(0)=g(0)=$ $1 / \sqrt{2 \pi}$, и $f^{(\alpha)}(\omega)=f(\alpha \omega), g^{(\alpha)}(\omega)=g(\alpha \omega)$.

Лемма 3.1 (о четырех пределах). Пусть $\widehat{P}_{t}^{(\alpha)}(\lambda)$ - однопараметрическая унитарная группа операторов в $L_{2}(\mathbb{R})$ с генератором $\widehat{N}_{\alpha}(\lambda)=\omega+\lambda\left|g^{(\alpha)}\right\rangle\left\langle g^{(\alpha)}\right| u$ $\widehat{\pi}_{T}=\mathscr{F}_{t \rightarrow \omega} I_{T}(t) \mathscr{F}_{\omega \rightarrow t}^{*}-$ семейство проекторов из $\mathscr{H}\left(L_{2}\right)(\mathbb{R})$. Тогда при $\alpha \rightarrow 0$ существуют предель
1) $\lim \int_{0}^{t} d s\left(g^{(\alpha)}, \widehat{P}_{s}^{(\alpha)}(\lambda) f^{(\alpha)}\right)=(2-i \lambda)^{-1}$
2) $w-\lim \int_{0}^{t} d s \widehat{P}_{s}^{(\alpha)}(\lambda) f^{(\alpha)}(\omega)=e^{i \omega t} \widetilde{I}_{(0, t)}(\omega)(1-i \lambda / 2)^{-1}$;
3) $w-\lim \left(g^{(\alpha)}, \widehat{P}_{t}^{(\alpha)}(\lambda) v\right)=(1-i \lambda / 2)^{-1} \mathscr{F}_{\omega \rightarrow t}^{*} v$;
4) $s-\lim \widehat{P}_{t}^{(\alpha)}(\lambda)=\exp \left\{i Z(\lambda) \widehat{\pi}_{(0, t)}\right\}=\widehat{P}_{t}(\lambda), \exp \{i Z(\lambda)\}=(2+i \lambda) /(2-i \lambda)$.

Рассмотрим семейство $\mathbf{H}_{\alpha}$ самосопряженных гамильтонианов (3.1), параметризованных функциональными аргументами операторов рождения и уничтожения $f^{(\alpha)}(\omega)$, $g^{(\alpha)}(\omega)$. Лемма 3.1 (см. [23]) обосновьвает предельньй переход в $(3.2)$ при $\alpha \rightarrow+0$. Пределы 1)-4) соответствуют предельным переходам в четырех составляющих решения (3.2), зависящих от $\alpha$. Подставляя 1)-4) в (3.2), получаем предельную унитарную группу $U_{t}=\exp \{i \mathbf{H} t\}=s-\lim _{\alpha \rightarrow 0} \exp \left\{i \mathbf{H}_{\alpha} t\right\}$ :

$$
\begin{aligned}
U_{t} h \otimes \psi(v)= & \int e^{i H(\lambda) t} d E_{\lambda} h \psi\left(e^{i Z(\lambda) \widehat{\pi}_{[0, t)}} e^{i \omega t} v+\frac{2 i}{2-i \lambda} \rho(\lambda) e^{i \Phi(\lambda)} \widetilde{I}_{[0, t)}\right) \\
& \times \exp \left\{\rho(\lambda) e^{-i \Phi(\lambda)} \frac{2 i}{2-i \lambda}\left(\widetilde{I}_{[0, t)}, e^{i \omega t} v\right)\right\}
\end{aligned}
$$

где $H(\lambda)=\nu(\lambda)+i \rho^{2} /(2-i \lambda)$. Обозначим через $W$ и $L$ операторы со спектральньми плотностями $(2+i \lambda) /(2-i \lambda)$ и $2 i /(2-i \lambda) \rho(\lambda) e^{i \Phi(\lambda)}$ соответственно. 
Тогда $2 i \rho(\lambda) e^{-i \Phi(\lambda)} /(2-i \lambda)-$ спектральная плотность оператора $-L^{*} W$, причем $W=(2+i K) /(2-i K)-$ преобразование Кэли самосопряженного оператора $2 K$, $L=2 i /(2-i K) R-$ плотно определенньй оператор такой, что $\operatorname{dom} L \supseteq \operatorname{dom} R, \mathrm{a}$ $H(\lambda)$ - спектральная функция оператора $i G=H_{0}-\frac{1}{4} L^{*} K L+\frac{i}{2} L^{*} L$. В дальнейшем изложении предполагается, что оператор $-G$ является генератором сильно непрерывной сжимающей однопараметрической полугрупшы $W_{t}=\exp \{-G t\}$, действующей в $\mathscr{H}$, причем

$$
D=\operatorname{dom} H \cap \operatorname{dom} L^{*} L \subseteq \operatorname{dom} G \subseteq \operatorname{dom} L, \quad G^{*} \varphi+G \varphi=L^{*} L \varphi \quad \forall \varphi \in D,
$$

где оператор $H=-H_{0}+\frac{1}{4} L^{*} K L$ симметричен на $D$. В этих обозначениях билинейная форма

$$
\mathbf{H}[g \otimes \psi(f), h \otimes \psi(v)]=\lim _{t \rightarrow+0} \frac{1}{i} \frac{d}{d t}\left(g \otimes \psi(f), U_{t} h \otimes \psi(v)\right),
$$

где $g, h \in D \subseteq \mathscr{H}, f, v \in \widetilde{W}_{2}^{1}(\mathbb{R})$, действует по правилу

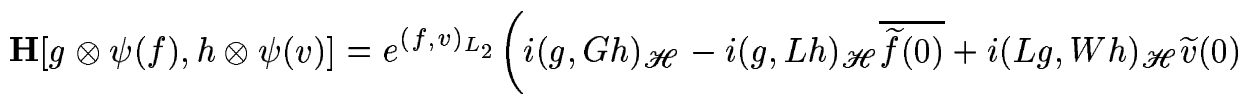

$$
\begin{aligned}
& \left.+(g, h)_{\mathscr{H}} \int d \omega \bar{f}(\omega) g(\omega) \omega+i(g,(I-W) h)_{\mathscr{H}} \overline{\widetilde{f}(0)} \widetilde{v}(0)\right)
\end{aligned}
$$

Билинейная форма (3.4) имеет регулярную диссипативную составляющую $\frac{i}{2} L^{*} L \otimes I$ и сингулярную в смысле [24], [25] составляющую

$$
-i L \otimes A^{+}\left(\frac{1}{\sqrt{2 \pi}}\right)+i L^{*} W \otimes A\left(\frac{1}{\sqrt{2 \pi}}\right)+i(I-W) \otimes A^{+}\left(\frac{1}{\sqrt{2 \pi}}\right) A\left(\frac{1}{\sqrt{2 \pi}}\right),
$$

обращающуюся в нуль на тотальном в $\mathbf{h}$ подмножестве

$$
\mathscr{D}_{0}=\left\{\Phi: \Phi=h \otimes \psi(v), v \in \widetilde{W}_{2}^{1}(\mathbb{R}), \widetilde{v}(0)=0, h \in D\right\}
$$

В то же время нетрудно видеть, что слабый предел последовательности $H_{\alpha}$ описывается билинейной формой, имеющей другие регулярную и сингулярную составляющие:

$$
\begin{aligned}
\lim _{\alpha \rightarrow 0} H_{\alpha}[g \otimes \psi(f), h \otimes \psi(v)]= & e^{(f, v)_{L_{2}}\left(\left(g, H_{0} h\right)_{\mathscr{H}}+(g, h)_{\mathscr{H}} \int \omega d \omega \bar{f}(\omega) g(\omega)\right.} \\
& \left.+(g, K h)_{\mathscr{H}} \overline{\widetilde{f}(0)} \widetilde{v}(0)+(g, R h)_{\mathscr{H}} \overline{\widetilde{f}(0)}-(R g, h)_{\mathscr{H}} \widetilde{v}(0)\right) .
\end{aligned}
$$

Здесь так же, как и в примере из второго раздела,

$$
r-\lim _{\alpha \rightarrow 0} \mathbf{H}_{\alpha} \neq w-\lim _{\alpha \rightarrow 0} \mathbf{H}_{\alpha}
$$

Рассмотрим функцию множества $u(s, t)=J_{s} U_{t-s} J_{t}^{*}$, где $J_{t}$ - унитарная однопараметрическая группа с генератором $\widehat{E}=\int d \omega \omega a^{+}(\omega) a(\omega)\left(\right.$ см. [7]), действующая в $\Gamma^{S}$ по правилу $J_{t} \psi(v)=\psi\left(e^{i \omega t} v\right)$. 
Учитывая, что $J_{t}^{*} \psi(v)=\psi\left(e^{-i \omega t} v\right)$, мы получаем из $(3.3)$

$$
\begin{aligned}
u(T) h \otimes \psi(v)= & \int e^{i H(\lambda) \operatorname{mes} T} d E_{\lambda} h \otimes \psi\left(e^{i Z(\lambda) \widehat{\pi}_{T}} v+i \rho(\lambda) e^{i \Phi(\lambda)} \frac{2}{2-i \lambda} \widetilde{I}_{T}\right) \\
& \times \exp \left\{i \rho(\lambda) e^{-i \Phi(\lambda)} \frac{2}{2-i \lambda}\left(\widetilde{I}_{T}, v\right)\right\} .
\end{aligned}
$$

Семейство коммутирующих операторов $u(T)$ удовлетворяет закону композиции $u\left(T_{1} \cup T_{2}\right)=u\left(T_{1}\right) u\left(T_{2}\right)$ для непересекающихся множеств, а дифференциал билинейной формы $(g \otimes \psi(f), u(0, t) h \otimes \psi(v))$ удовлетворяет слабому квантовому стохастическому дифференциальному уравнению [2]

$$
d(h \otimes \psi(v), u(0, t) h \otimes \psi(v))=i\left(h \otimes \psi(v), u(0, t) H\left(d t_{+}\right) h \otimes \psi(v)\right),
$$

где

$$
\begin{gathered}
i H(T)=M(T)=\int_{T} d t J_{t}(\mathbf{H}-\widehat{E} \otimes I) J_{t}^{*}, \\
M(T)=-G \otimes \operatorname{mes} T+L \otimes A^{+}(T)-L^{*} W \otimes A(T)+(W-I) \otimes \Lambda(T) .
\end{gathered}
$$

Таким образом, имеет место следующее утверждение.

ТЕорема 3.2. С точностью до унитарного преобразования $J_{t}$ семейство решений уравнения Шрёдингера с гамильтонианом (3.1) сильно сходится в $\mathbf{h} к$ решению $u(t)$ стохастического уравнения (3.6) $u(0, t)=s-\lim _{\alpha \rightarrow 0} U_{t}^{(\alpha)} J_{t}^{*}$.

Как мы увидим ниже, неожиданным обстоятельством является то, что естественная область определения генератора группы $U_{t}$ (множество значений резольвенты) не содержит функций, на которых корректно определена билинейная форма (3.4): Фурье-образы фоковских компонент резольвенты имеют скачки амплитуды и фазы при смене знака аргументов.

4. Сюрпризы резольвенты. Рассмотрим вектор $\Phi \in \mathbf{h}=\mathscr{H} \otimes \Gamma^{S}\left(L_{2}(\mathbb{R})\right)$ :

$$
\begin{gathered}
\Phi=R_{\mu} h \otimes \psi(v)=\int_{0}^{\infty} d t e^{-\mu t} U_{t} h \otimes \psi(v)=\left\{\Phi_{n}(\omega)\right\} \\
\Phi_{n}(\cdot): \mathbb{R}^{n} \rightarrow \mathscr{H}, \quad \omega=\left\{\omega_{1}, \ldots, \omega_{n}\right\}
\end{gathered}
$$

с компонентами (3.3)

$$
\begin{gathered}
\Phi_{n}(\omega)=\int_{0}^{\infty} d t \exp \left\{-\mu(G+t)-L^{*} W \int_{0}^{t} \widetilde{v}(-\tau) d \tau\right\} \varphi_{n, t}(\omega), \\
\varphi_{n, t}(\omega)=\prod_{1}^{n}\left((W-1) \pi_{[0, t)} e^{i \omega_{k} t} v\left(\omega_{k}\right)+e^{i \omega_{k} t} v\left(\omega_{k}\right)+L \widetilde{I}_{[0, t)}\left(\omega_{k}\right)\right) h
\end{gathered}
$$

где $L, W, G$ - введенные вьше коммутирующие операторы со спектральными плотностями

$$
L(\lambda)=2 i \rho(\lambda) e^{-i \Phi(\lambda)}(2-i \lambda)^{-1}, \quad W(\lambda)=e^{i Z(\lambda)}, \quad G(\lambda)=-i \nu(\lambda)+\frac{\rho(\lambda)^{2}}{(2-i \lambda)} .
$$


Обозначим через $\widetilde{\varphi}_{n, t}$ Фурье-прообраз функции $\varphi_{n, t}$ по переменным $\omega_{k}$

$$
\widetilde{\varphi}_{n, t}(\tau)=\prod_{1}^{n}\left((W-I) I_{[0, t)}\left(\tau_{k}\right) \widetilde{v}\left(\tau_{k}-t\right)+\widetilde{v}\left(\tau_{k}-t\right)+L I_{[0, t)}\left(\tau_{k}\right)\right) h
$$

где $\tau=\left\{\tau_{1}, \ldots, \tau_{n}\right\}$. Пусть $\mathscr{K}$ - подмножество в $\{1, \ldots, n\}$ и $\mathscr{K}^{a}-$ его дополнение. Положим

$$
P_{\mathscr{K}, t}^{(n)}(\tau)=\prod_{k \in \mathscr{K}}\left((W-I) \widetilde{v}\left(\tau_{k}-t\right)+L\right) I_{[0, t)}\left(\tau_{k}\right) \in \mathscr{B}(\mathbf{h}) .
$$

Тогда

$$
\widetilde{\varphi}_{n, t}(\tau)=\sum_{\mathscr{K}}\left(P_{\mathscr{K}, t}^{(n)}(\tau) \prod_{m \in \mathscr{K}^{a}} \widetilde{v}\left(\tau_{m}-t\right)\right) h
$$

Нетрудно видеть, что функции $P_{\mathscr{K}, t}^{(n)}(\tau)$ имеют разрьвы первого рода при смене знака переменных $\tau_{k}$ :

$$
\begin{aligned}
\lim _{\tau_{k} \rightarrow-0} P_{\mathscr{K}, t}^{(n)}(\tau) & =I_{\mathscr{K}^{a}}(k) P_{\mathscr{K}, t}^{(n)}(\tau), \\
\lim _{\tau_{k} \rightarrow+0} P_{\mathscr{K}, t}^{(n)}(\tau) & =I_{\mathscr{K}^{a}}(k) P_{\mathscr{K}, t}^{(n)}(\tau)+((W-I) \widetilde{v}(-t)+L) P_{\mathscr{K} \backslash\{k\}, t}^{(n-1)}(\tau) .
\end{aligned}
$$

Поэтому (4.2) дает следующее значение для разности подстановок:

$$
\left.P_{\mathscr{K}, t}^{(n)}(\tau)\right|_{\tau_{k}=+0} ^{\tau_{k}=-0}=-((W-I) \widetilde{v}(-t)+L) P_{\mathscr{K} \backslash\{k\}, t}^{(n-1)}(\tau) I_{\mathscr{K}}(k) .
$$

Вычислим скачок $\widetilde{\varphi}_{n, t}(\tau)$ при изменении знака $\tau_{k}$. Заметим, что

$$
\lim _{\tau_{k} \rightarrow-0} \widetilde{\varphi}_{n, t}(\tau)=\widetilde{v}(-t) \widetilde{\varphi}_{n-1, t}\left(\tau_{1}, \ldots, \tau_{k-1}, \tau_{k+1}, \ldots, \tau_{n}\right) .
$$

Учитьвая (4.1) и (4.3), находим значения скачка амплитуды и фазы функций из области определения инфинитезимального оператора группы $U_{t}$

$$
\lim _{\tau_{k} \rightarrow+0} \widetilde{\varphi}_{n, t}(\tau)=W \lim _{\tau_{k} \rightarrow-0} \widetilde{\varphi}_{n, t}(\tau)+L \widetilde{\varphi}_{n-1, t}\left(\tau_{1}, \ldots, \tau_{k-1}, \tau_{k+1}, \ldots, \tau_{n}\right) .
$$

Обозначим через $\mathscr{D}_{W, L}=D \otimes \Gamma^{S}\left(\widetilde{W}_{2}^{1}(\mathbb{R} \backslash\{0\})\right)$ векторное подпространство в $\mathbf{h}$, элементы которого удовлетворяют условию (4.4), а через $A\left(\delta_{ \pm}\right), \Lambda\left(\delta_{ \pm}\right), \widehat{N}$ обозначим операторы, действующие на фоковские векторы по правилам

$$
\begin{aligned}
\left(\Phi, \Lambda\left(\delta_{ \pm}\right) \Psi\right) & =\left.\lim _{\varepsilon \rightarrow+0} \sum_{1}^{\infty} \frac{1}{n !} \sum_{k=1}^{n} \int_{(\mathbb{R} \backslash\{0\})^{n-1}} \prod_{m \neq k} d \tau_{m}\left(\widetilde{\Phi}_{n}, \widetilde{\Psi}_{n}\right) \mathscr{H}\right|_{\tau_{k}= \pm \varepsilon}, \\
\mathscr{F}_{\omega \rightarrow \tau}\left(A\left(\delta_{ \pm}\right) \Psi\right)_{n}(\tau) & =\left.\lim _{\varepsilon \rightarrow+0} \sum_{k=1}^{n} \widetilde{\Psi}_{n+1}(\tau)\right|_{\tau_{k}= \pm \varepsilon}, \quad \widehat{N} \Psi_{n}(\omega)=n \Psi_{n}(\omega) .
\end{aligned}
$$

В этих обозначениях краевое условие (4.4) в $\Gamma^{S}$ принимает вид

$$
(\widehat{N}+1)^{-1}\left(I \otimes A\left(\delta_{+}\right)-W \otimes A\left(\delta_{-}\right)\right) \Psi=L \otimes I \Psi
$$


Оно накладьвается на компоненты фоковского вектора $\Psi_{1}, \Psi_{2}, \ldots$ и является обобщением условия (2.2) для фоковского пространства.

Покажем, что оператор

$$
\widehat{H}=i G \otimes I+I \otimes \widehat{E}+i L^{*} W \otimes A\left(\delta_{-}\right), \quad \widehat{E}=\int_{\mathbb{R} \backslash\{0\}} d \tau a^{+}(\tau) a(\tau) i \partial_{\tau},
$$

симметричен на $\mathscr{D}_{W, L}$. Пусть $\Phi, \Psi \in \mathscr{D}_{W, L}$ и пусть $B-$ эрмитов оператор, $\operatorname{dom} B \otimes I \supseteq$ $\mathscr{D}_{W, L}$. Формула интегрирования по частям дает тождество, в котором разность подстановок выражается через операторы $\Lambda\left(\delta_{ \pm}\right)$

$$
\begin{aligned}
& (\Phi, B \otimes \widehat{E} \Psi)-(B \otimes \widehat{E} \Phi, \Psi)=i\left(\Phi, B \otimes\left(\Lambda\left(\delta_{-}\right)-\Lambda\left(\delta_{+}\right)\right) \Psi\right) \\
& \quad=\left.i \sum_{1}^{\infty} \frac{1}{n !} \sum_{k=1}^{n} \int_{(\mathbb{R} \backslash\{0\})^{n-1}} \prod_{m \neq k} d \tau_{m}\left(\widetilde{\Phi}_{n}(\tau), B \widetilde{\Psi}_{n}(\tau)\right)_{\mathscr{H}}\right|_{\tau_{k}=+0} ^{\tau_{k}=-0}
\end{aligned}
$$

Используя краевое условие (4.5) для функций $\widetilde{\varphi}_{n}$ и $\widetilde{\psi}_{n}$, находим разность подстановок значения интеграла (4.7):

$$
\begin{aligned}
& i\left(\Phi, B \otimes\left(\Lambda\left(\delta_{+}\right)-\Lambda\left(\delta_{-}\right)\right) \Psi\right)=i\left(\Phi,\left(W^{*} B W-B\right) \Lambda\left(\delta_{-}-\Psi\right)\right. \\
& \quad+i(L \Phi, B L \Psi)+i\left(W A\left(\delta_{-}\right) \Phi, B L \Psi\right)+i\left(L \Phi, B W A\left(\delta_{-}\right) \Psi\right) .
\end{aligned}
$$

В частности, при $B=I$ (4.8) упрощается:

$i\left(\Phi, I \otimes\left(\Lambda\left(\delta_{+}\right)-\Lambda\left(\delta_{-}\right)\right) \Psi\right)=i\left(\Phi, L^{*} L \Psi\right)-\left(i L^{*} W \otimes A\left(\delta_{-}\right) \Phi, \Psi\right)+\left(\Phi, i L^{*} W \otimes A\left(\delta_{-}\right) \Psi\right)$.

Учитывая, что $i G-i L^{*} L=(i G)^{*}$, мы получаем отсюда тождество, означающее симметричность оператора $\widehat{H}$ на $\mathscr{D}_{W, L}$ :

$$
\begin{aligned}
(\Phi, \widehat{H} \Psi)= & ((I \otimes \widehat{E}) \Phi, \Psi)+\left(\Phi,\left\{i G \otimes I+i L^{*} W \otimes A\left(\delta_{-}\right)\right\} \Psi\right) \\
& -i\left(\Phi, I \otimes\left(\Lambda\left(\delta_{+}\right)-\Lambda\left(\delta_{-}\right)\right) \Psi\right) \\
= & ((I \otimes \widehat{E}) \Phi, \Psi)+\left(\Phi,(i G)^{*} \otimes I \Psi\right)+\left(i L^{*} W \otimes A\left(\delta_{-}\right) \Phi, \Psi\right)=(\widehat{H} \Phi, \Psi) .
\end{aligned}
$$

Вычислим действие производящего оператора групшы $U_{t}$ на множестве значений резольвенты. Пусть $\Psi \in \mathbf{h}, \Phi=R_{\mu} h \otimes \psi(v)$. Из определения производящего оператора имеем

$$
\begin{aligned}
(\Psi, \widehat{H} \Phi)= & \lim _{s \rightarrow+0} \frac{1}{i} \frac{d}{d s}\left(\Psi, U_{s} \Phi\right)=\frac{1}{i} \int_{0}^{\infty} d t \sum_{n=0}^{\infty} \frac{1}{n !} \int_{(\mathbb{R} \backslash\{0\})^{n}} d \tau \\
& \times\left(\widetilde{\psi}_{n}(\tau), \frac{d}{d s} e^{-(G+\mu) t-G s-i L^{*} W \int_{0}^{t+s} \widetilde{v}(-\tau) d \tau} \widetilde{\varphi}_{n, t+s}(\tau)\right)_{\left.\mathscr{H}\right|_{s=0}}
\end{aligned}
$$

Заметим, что функции $\widetilde{\varphi}_{n, t}(\tau)$ зависят от разностей $\tau_{k}-t$. Поэтому

$$
\frac{d}{d t} \widetilde{\varphi}_{n, t}(\tau)=-\sum_{k=1}^{n} \frac{\partial}{\partial \tau_{k}} \widetilde{\varphi}_{n, t}(\tau)=i \widehat{E}_{\varphi_{n, t}}(\tau)
$$

С другой стороны, из определения оператора $A\left(\delta_{-}\right)$следует

$$
A\left(\widetilde{\left.\delta_{-}\right) \varphi_{n, t}}(\tau)=n \widetilde{v}(-t) \varphi_{n-1, t}(\tau)\right.
$$


Теперь, учитывая определение (4.9) и тождества (4.10) и (4.11), получаем

$$
\begin{aligned}
& (\Psi, \widehat{H} \Phi)_{\mathbf{h}}=\int_{0}^{\infty} d t\left(\psi_{0}, i G e^{-(G+\mu) t-i L^{*} W \int_{0}^{t} \widetilde{v}(-\tau) d \tau} h\right)_{\mathscr{H}}+\int_{0}^{\infty} d t \sum_{n=1}^{\infty} \frac{1}{n !} \int_{(\mathbb{R} \backslash\{0\})^{n}} d \tau \\
& \quad \times\left(\widetilde{\psi}_{n}(\tau), e^{-(G+\mu) t-i L^{*} W \int_{0}^{t} \widetilde{v}(-\tau) d \tau}\left(i G+i L^{*} W \widetilde{v}(-t)+i \sum_{k=1}^{n} \frac{\partial}{\partial \tau_{k}}\right) \widetilde{\varphi}_{n, t}(\tau)\right)_{\mathscr{H}} \\
& =\left(\Psi,\left\{i G+i L^{*} W \otimes A\left(\delta_{-}\right)+I \otimes \widehat{E}\right\} \Phi\right)_{\mathbf{h}}
\end{aligned}
$$

т.е. производящий оператор $\widehat{H}$ группы $U_{t}$ имеет вид (4.6). Таким образом, доказана следующая теорема.

Tеорема 4.1. Симметричный на $\mathscr{D}_{W, L}$ оператор $\widehat{H}=i G \otimes I+I \otimes \widehat{E}+i L^{*} W \otimes A\left(\delta_{-}\right)$ $\left(G=i H+\frac{1}{2} L^{*} L, H=\frac{1}{4} L^{*} K L-H_{0}\right)$ является производящим оператором унитарной однопараметрической группь $U_{t}$.

Важно отметить, что проверка симметричности не предполагает коммутативность операторов $L, G, W$ и легко распространяется на операторы вида

$$
\widehat{H}=i G+I \otimes \widehat{E}+i \sum_{l, m} L_{l}^{*} W_{l, m} \otimes A_{m}\left(\delta_{-}\right)
$$

с краевым условием

$$
(\widehat{N}+1)^{-1}\left(I \otimes A_{l}\left(\delta_{+}\right)-\sum_{m} W_{l, m} \otimes A_{m}\left(\delta_{-}\right)\right) \Psi=L_{l} \otimes I \Psi,
$$

где $W=\left\{W_{l, m}\right\}-(M \times M)$-матрица с коэффициентами из $\mathscr{B}(\mathscr{H})$ такая, что $W^{*} W=I$, a $\left\{A_{m}(g): g \in L_{2}(\mathbb{R}), 1 \leqslant m \leqslant M\right\}$ - операторы уничтожения в $\Gamma^{S}\left(L_{2}\left(\mathbb{R}^{M}\right)\right)$, коммутирующие при различных $l$.

5. Уравнение марковской эволюции. В заключение опишем вьвод уравнения марковской эволюции из задачи Коши для уравнения Шрёдингера

$$
\frac{d}{d t} \Psi(t)=\left(-G+i I \otimes \widehat{E}-\sum_{l, m} L_{l}^{*} W_{l, m} \otimes A_{m}\left(\delta_{-}\right)\right) \Psi(t)
$$

с краевым условием (4.13). Пусть $B$ - эрмитов оператор из $\mathscr{B}(\mathscr{H})$ и $h, g \in D$. Рассмотрим уравнение для среднего значения

$$
\left(g, P_{t}(B) h\right)_{\mathscr{H}}=\left(U_{t} g \otimes \Psi(0)|B \otimes I| U_{t} h \otimes \Psi(0)\right)_{\mathbf{h}} .
$$

Из (4.12) имеем

$$
\begin{aligned}
\frac{d}{d t}\left(g, P_{t}(B) h\right) \mathscr{H}= & -\left(\left(G+\sum_{l, m} L_{l}^{*} W_{l, m} A_{m}\left(\delta_{-}\right)\right) U_{t} g \otimes \Psi(0)|B \otimes I| U_{t} h \otimes \Psi(0)\right) \\
& -\left(U_{t} g \otimes \Psi(0)|B \otimes I|\left(G+\sum_{l, m} L_{l}^{*} W_{l, m} A_{m}\left(\delta_{-}\right)\right) U_{t} h \otimes \Psi(0)\right) \\
& \left.+\left(U_{t} g \otimes \Psi(0)\left|B \otimes\left(\Lambda\left(\delta_{+}\right)-\Lambda\left(\delta_{-}\right)\right)\right| U_{t} h \otimes \Psi(0)\right)\right) .
\end{aligned}
$$


Теперь воспользуемся тождеством (4.8), которое в данном случае записьвается в виде

$$
\begin{aligned}
& \left(\Phi, B \otimes\left(\Lambda\left(\delta_{+}\right)-\Lambda\left(\delta_{-}\right)\right) \Psi\right)=\sum_{l, m}\left(\Phi,\left(W_{l, m}^{*} B W_{l, m}-B\right) \Lambda_{m}\left(\delta_{-}\right) \Psi\right) \\
& \quad+\sum_{l}\left(L_{l} \Phi, B L_{l} \Psi\right)+\sum_{l, m}\left(\left(W_{l, m} A_{m}\left(\delta_{-}\right) \Phi, B L_{l} \Psi\right)+\left(L_{l} \Phi, B W_{l, m} A_{m}\left(\delta_{-}\right) \Psi\right)\right) .
\end{aligned}
$$

Учитьвая, что $A\left(\delta_{-}\right) \Psi(0)=0, \Lambda\left(\delta_{-}\right) \Psi(0)=0$, получаем из $(5.1),(5.2)$

$$
\left.\frac{d}{d t}\left(g, P_{t}(B) h\right)_{\mathscr{H}}\right|_{t=0}=(g, \mathscr{L}(B) h)=-(G g, B h)-(g, B G h)+\sum_{l}\left(L_{l} g, B L_{l} g\right) .
$$

Таким образом, получено производящее отображение $\mathscr{L}(\cdot)$ в форме Линдблада для уравнения марковской эволюции

$$
\frac{d}{d t} P_{t}(B)=\mathscr{L}\left(P_{t}(B)\right), \quad \mathscr{L}(B)=-G^{*} B-B G+\frac{1}{2} \sum_{l} L_{l}^{*} B L_{l}, \quad G=i H+\frac{1}{2} \sum_{m} L_{m}^{*} L_{m} .
$$

Московский государственный университет

Поступило

им. М.В. Ломоносова

11.12 .96

\section{СПИСОК ЦИТИРОВАННОЙ ЛИТЕРАТУРЫ}

[1] Lindblad G. On the generators of quantum dynamical semigroups // Comm. Math. Phys. 1976. V. 48. № 2. P. 119-130.

[2] Hudson R. L., Parthasarathy K. R. Quantum Ito's formula and stochastic evolutions // Comm. Math. Phys. 1984. V. 93. №3. P. 301-323.

[3] Parthasarathy K.R. An introduction to quantum stochastic calculus. Basel: Birkhauser, 1992.

[4] Meyer P. A. Quantum probability for probabilists. Lecture Notes in Math. V. 1338. Berlin: Springer Verlag, 1993.

[5] Davies E. B. Quantum theory of open systems. London: Acad. Press, 1976.

[6] Gorini V., Kossakovsky A., Sudarshan E. C. G. Completely positive dynamical semigroups of $n$-level systems // J. Math. Phys. 1976. V. 17. № 3. P. 821-825.

[7] Gardiner C.W., Collett M. J. Input and output in damped quantum systems: quantum statistical differential equations and the master equation // Phys. Rev. A. 1985. V. 31. P. 3761-3774.

[8] А.Н. Колмогоров, С.П. Новиков (ред. ) Квантовые случайные процессы и открытые системы. Сер. «Математика. Новое в зарубежной науке». Т. 42. М.: Мир, 1988.

[9] Хасьминский Р. З. Эргодические свойства возвратных диффузий и стабилизация задачи Коши для параболического уравнения // Теория вероятн. и ее применения. 1960. Т. 5. № 1. C. 196-214.

[10] Ichihara K. Explosion problems for symmetric diffusion processes // Lecture Notes in Math. 1986. V. 1203. P. 75-89.

[11] Чеботарев А. М. Необходимые и достаточные условия консервативности динамических полугрупп // Итоги науки и техн. Соврем. пробл. матем. Новейшие достижения. Т. 36. М.: ВИНИТИ, 1990. С. 149-184.

[12] Чеботарев А. М. Достаточные условия для консервативности минимальной динамической полугруппы // Матем. заметки. 1992. Т. 52. №4. С. 112-122. 
[13] Chebotarev A. M., Fagnola F., Frigerio A. Towards a stochasic Stone's theorem // Stochastic patial differential equations and applications. Pitman Res. Notes Math. Ser. V. 268. Harlow: Longman Sci. Tech., 1992. P. 86-97.

[14] Chebotarev A. M., Fagnola F. Sufficient conditions for conservativity of quantum dynamical semigroups // J. Funct. Anal. 1993. V. 113. № 1. P. 131-153.

[15] Holevo A.S. On conservativity of covariant dynamical semigroups // Rep. Math. Phys. 1993. V. 33. P. $95-100$.

[16] Bhat B. V.R., Parthasarathy K.R. Markov dilations of non-conservative dynamical semigroups and a quantum boundary theory // Ann. Inst. H. Poincaré. Probab. Statist. 1995. V. 31. № 4. P. 601-651.

[17] Holevo A.S. On the structure of covariant dynamical semigroups // J. Funct. Anal. 1995. V. 131. P. 255-278.

[18] Чеботарев А. М., Гарсия Х. К., Гезада Р. Б. Об уравнении Линдблада с неограниченными переменными коэффициентами // Матем. заметки. 1997. Т. 61. №1. С. 125-140.

[19] Chebotarev A. M. Minimal solutions in classical and quantum probability // Quantum Probability and Related Topics. VII / ed. L. Accardi. Singapore: World Scientific, 1992. P. 79-91.

[20] Fagnola F. Characterization of isometric and unitary weakly differentiable cocycles in Fock space // Quantum Probability and Related Topics. VIII. Preprint № 358. Trento: UTM, 1993. P. 143-164.

[21] Bhat B. V. R., Fagnola F., Sinha K. B. On quantum extensions of semigroups of Brownian motions on a half-line // Russian J. Math. Phys. 1996. V. 4. №1. P. 13-28.

[22] Journé J. L. Structure des cocycles markoviens sur l'espace de Fock // Probab. Theory Related Fields. 1987. V. 75. P. 291-316.

[23] Чеботарев А. М. Симметризованная форма стохастического уравнения Хадсона-Партасарати // Матем. заметки. 1996. Т. 60. №5. С. 726-750.

[24] Кошманенко В. Д. Возмушения самосопряженных операторов сингулярными билинейными формами // Укр. матем. ж. 1989. Т. 41. № 1. С. 3-18.

[25] Кошманенко В. Д. Сингулярные билинейные формы в теории возмущений самосопряженных операторов. Киев: Наукова думка, 1993.

[26] Albeverio S., Karwowski W., Koshmanenko V. Square powers of singularly perturbed operators // Math. Nachr. 1995. V. 173. P. 5-24.

[27] Като Т. Теория возмущений линейных операторов. М.: Наука, 1972.

[28] Березин Ф. А. Метод вторичного квантования. М.: Наука, 1986. 